\title{
Practice Patterns of Audiologists Involved in Assessment and Management of Vestibular Disorders: An Indian Cross- Sectional Survey
}

\author{
Kaushalendra kumar* \\ Ashna Elizabath Shaju, \\ Priya Karimuddanahalli Premkumar, \\ Anupriya Ebenezer, \\ Ritik Roushan
}

\begin{abstract}
Background: As the incidence of vestibular disorders continues to rise, the role of the audiologists in assessing and providing effective intervention has become increasingly important. To date, there have been no studies investigating the practice patterns of Indian audiologists towards the assessment and management of vestibular disorders.

Method: An electronic survey consisting of 29 questions across demographics, vestibular assessment, and management emailed to 704 members of the Indian Speech and Hearing Association (ISHA). Additionally, the questionnaire was circulated to audiologists through social media groups. The participants were provided with a two weeks' time limit for completing the survey.

Results: 704 participants were contacted to take part in the survey, and only 243 responses were received resulting in a 34.66 $\%$ response rate. Among the 243 participants who responded, 127 participants were currently not involved in the assessment and/or management of vestibular disorders in India. The responses were analyzed descriptively according to each section of the questionnaire. The study also sheds light on vestibular assessment, rehabilitation trends, and perceived barriers/facilitators among Indian audiologists.
\end{abstract}

Conclusion: We observed major discrepancies between practice patterns of audiologists in India. Like any other developing nation, India has challenges of its own. Proper planning in terms of resource allocation is needed to address the barriers in access to instrumentation, and limited workforce.

Keywords: Awareness; assessment; management; audiologist; Vestibular; Severity.

Associate Professor, Department of audiology and speech language pathology, Kasturba medical college Mangalore, Manipal academy of higher education, Manipal, Karnataka, India

Associate Professor, Department of audiology and speech language pathology, Kasturba medical college Manipal academy of higher education, India. E-mail: kaushalendra.kumar@manipal.edu Phone: +9164699960

Paper submitted on April 23, 2021; and Accepted on July 16, 2021 


\section{INTRODUCTION}

The vestibular, visual, and proprioceptive systems function in synergy to bring about postural stability and visual equilibrium ${ }^{1}$. The presence of a vestibular disorder disrupts this synergistic functioning, especially the functioning of the vestibular system ${ }^{2}$. Several causes such as an underlying disease, trauma, vestibulotoxic medications, and advancements in age can result in damage to the vestibular system and are often associated with a history of dizziness and hearing loss ${ }^{1,2}$. The global incidence of vestibular problems such as dizziness, vertigo, and imbalance are reported to be about $15-20 \%$ and increases to $40 \%$ among those individuals ages 40 and above ${ }^{1}$. Also, studies ${ }^{2}$ have indicated that vertigo and dizziness are the greatest contributors to the burden of disability in the aged population. Some of the common aetiologies of vestibular disorders include, but are not limited to benign paroxysmal positional vertigo (BPPV), Meniere's disease, vestibular neuritis, labyrinthitis ${ }^{3}$, nystagmus, nausea, and ataxia ${ }^{4}$. Among these, the disorders of the inner ear are thought to contribute to most of the dizziness cases, at a rate of approximately $85 \%{ }^{5}$. Because such a high percentage of dizziness cases are related to a dysfunction within the inner ear system. Hence, it is crucial to understand the clinical practices of audiologists towards the assessment and management of vestibular disorders. However, only a handful of western studies ${ }^{6-8}$ have focused on investigating the current clinical practices of audiologists in assessing and managing vestibular disorders.

The Indian subcontinent poses a challenge that is different from that of the western scenario. Some of the challenges faced by the Indian health care sector include dense population, limited trained workforce, unequal distribution of resources between rural and urban provinces, and service quality. Due to the reasons mentioned above, assessment and management of vestibular disorders may be particularly challenging within the Indian public health sector. To date, there have been no studies investigating the practice patterns of Indian audiologists towards the assessment and management of vestibular disorders. The current study, therefore, aimed to explore the current practices of Audiologists in India towards the assessment and management of vestibular disorders. It is hoped that the data obtained from this survey will provide insight into the specific areas of vestibular practice that need additional formal education and/or clinical training for practicing audiologists.

\section{MATERIALS AND METHODS}

Study design: We adopted a descriptive cross-sectional design with non-randomized convenient sampling. The study was carried out at the Department of Audiology and Speech-Language Pathology, Kasturba Medical College, Mangalore, between the years of 2018 to 2020 .

Participants: Before the recruitment of participants, the participants of the survey were audiologists (clinical or academic) who were currently practicing in India. All the participants had a minimum qualification of bachelor's degree in Audiology and Speech-Language Pathology (BASLP) from a University recognized by the University Grants Commission (UGC) of India, and the Rehabilitation Council of India (RCl). Those participants working only as Speech-Language Pathologists were excluded from the survey.

Survey questionnaire: We began by constructing a survey questionnaire based on existing clinical practice studies 6-8. The questionnaire was content validated by 5 audiologists (3 practicing clinicians and 2 academicians). An electronic version of the questionnaire consisting of 29 questions categorized into three main sections (demographics, vestibular assessment, and vestibular management) was created using Google Forms. The final version of the questionnaire can be found in Figure 1.

Survey: The electronic version of the questionnaire was sent out to 704 members of the Indian Speech and Hearing Association (ISHA) through email. Additionally, the questionnaire was circulated to professional (audiology) groups on social media. The participants were provided with a two weeks' time limit for completing the survey.

\section{RESULTS AND DISCUSSION}

The current study aimed to investigate the national practices in the assessment and management of vestibular disorders among Audiologists in India. We carried out a nationwide survey probing three main domains of demographics, vestibular assessment, and vestibular rehabilitation.

Survey Response Rate: Initially, 704 participants were contacted to take part in the survey, and only 243 responses were received resulting in a $34.66 \%$ response rate. Among the 243 participants who responded, and/or management of vestibular disorders in India. Hence, their responses were discarded and only 116 responses were descriptively analyzed in sections 2 , and 3 of the survey.

\section{Section 1: Participant Demographics}

Education: Among the 243 participants who completed the demographic details section, 94 (39\%) had a bachelor's degree in audiology and speech-language pathology, and 149 (61\%) participants had a master's degree or higher.

Work settings: The study participants were from varied work settings. $37 \%$ of them reported that they were working in a private clinic setup, $31 \%$ in a hospital setup, $25 \%$ at University/ educational institution, and $7 \%$ at rehabilitation units.

Years of experience: For the years of practice, 19\% of the participants had less than 1 year, almost $50 \%$ of the participants had 1-5 years of experience, $20 \%$ had $6-10$ years, $5 \%$ for $10-15$ years, and $6 \%$ of the participants had more than 15 years. Similar findings have been reported in a practice pattern survey from South Africa ${ }^{7}$, where the 
majority of audiologists had a clinical experience of 0-5 years in the assessment and management of vestibular dysfunctions. In addition to the demographic information, a large portion of the participants reported that they were not involved in the assessment and/or management of vestibular disorders. $51 \%$ of the participants reported that they did not perform vestibular assessment ${ }^{9}$. The remaining $49 \%$ of participants, who reported that they perform the vestibular assessment were asked about how frequently they performed a vestibular assessment. $20 \%$ of them 'always' performed a vestibular assessment, $18 \%$ reported 'rarely,' and $11 \%$ of them did it 'mostly but not always $48 \%$ of the participants reported that they were not involved in vestibular rehabilitation. However, $22 \%$ of the participants reported that they were 'rarely' involved in management, $16 \%$ of them did it 'mostly but not always,' and $14 \%$ of them were 'always' involved in management. These national trends are a reflection of international practice trends; a similar trend was reported by Seedat et $\mathrm{al}^{7}$ among practicing audiologists in South Africa. Another study ${ }^{6}$ carried out in the United States between 2003 to 2014 report similar trends with $58 \%$ - $65 \%$ of audiologists not being involved in vestibular assessment/ management.

\section{Performing Vestibular Assessment and Management}

Here onwards, only those (116) participants who reported that they were involved in vestibular assessment and management. Among these 116 participants, 40\% had < one-year experience in vestibular practices (assessment and management), $42 \%$ of them had $1-5$ years, $13 \%$ had $6-10$ years, $4 \%$ had $10-15$ years, and a mere $1 \%$ above 15 years of experience.

Source of Training in Vestibular Practices: 59\% of participants reported that they received their training in vestibular assessment and management at their graduate or post-graduate level courses. $26 \%$ participants from special workshops or seminars, $6 \%$ from previous work settings and by self-learning, and $3 \%$ reported that they did not receive any training.

Section 2: Vestibular assessment: We began by asking the participants about the number of assessments that they performed monthly. $62 \%$ of the participants performed $<10$ assessments, 32\% 10-50, and less than $5 \%$ of the participants reported that they performed $>50$ assessments. We followed this up by asking them, where do they get their vestibular assessment referrals from? For this question, $83 \%$ of the participants reported that their source of referral was from an otorhinolaryngologist, $13 \%$ reported neurologists, $2 \%$ from general physicians, and the rest $(<2 \%)$ did not receive any referrals.

Screening practices: When asked about how frequently they performed vestibular screening, 34\% of participants reported that they performed screening 'always,' $27 \%$ performed 'mostly but not always,' $31 \%$ participants 'rarely' performed vestibular screening, and $8 \%$ reported that they did not screen for vestibular disorders. Following this, we asked our participants what were their choice of tools to screen for vestibular disorders? A wide variety of tools were reported by our participants, and their responses have been depicted in Figure 1. As shown in figure $1,44 \%$ of participants reported that the Dizziness Handicap Inventory (DHI) ${ }^{9}$ was their choice of subjective tool, $32 \%$ of participants reported Vertigo Handicap Questionnaire $(\mathrm{VHQ})^{10}, 7 \%$ reported Vestibular Disorders Activities of Daily Living (VADL) ${ }^{11}, 13 \%$ reported Vertigo Symptom Scale (VSS) ${ }^{12}$, and $5 \%$ reported others. Out of the 116 participants, 16 participants chose a combination of 2 or more screening tools.

\section{Objective Assessment of Vestibular Disorders}

We followed up by asking our participants, the choice of objective assessment tests of our participants has been shown in Figure 2. As shown in figure 2, 39\% of participants reported that they used Cervical Vestibular Evoked Myogenic Potentials (cVEMP) for objective assessment of vestibular disorders, $25 \%$ of participants reported Videonystagmography (VNG), 22\% reported the use of Ocular Vestibular Evoked Myogenic Potentials (oVEMP), 10\% utilized Electronystagmography (ENG), and $4 \%$ for reported Video Head Impulse Test (VHIT). Our participants also reported a combination of objective tests as a part of the test battery. 16 participants reported a combination of two objective tests (cVEMP and oVEMP), 25 participants reported a combination of three or more objective tests as a part of their test battery for evaluating vestibular disorders.

Test battery approach: We asked our participants how often they considered using a test battery approach towards the assessment of vestibular disorders, also, what were the tests included in their battery? A majority $(44 \%)$ of our participants reported that they 'always' used a test battery for the assessment of vestibular disorders. $34 \%$ reported 'sometimes', $15 \%$ reported that they 'could not say,' and $7 \%$ mentioned they 'did not use' a test battery for vestibular assessments. The constituents of the vestibular test battery used by the participants have been depicted in Figure 3.

Section 3: Vestibular Management: We asked our participants their opinion on the statement 'Audiologists have a major role in vestibular rehabilitation.' $48 \%$ of our participants 'agreed' to the statement, $38 \%$ had a 'neutral' opinion $10 \%$ of our participants 'disagreed,' and $2 \%$ of participants said they 'could not say.' We followed up with questions on referral sources and their frequency. $79 \%$ of the participants reported that they receive less than 10 referrals to perform vestibular rehabilitation, $14 \%$ reported that they would receive about 10-15 referrals, $6 \%$ received about $50-150$ referrals, and none of the participants received more than 150 referrals. Regarding the source of referrals, $69 \%$ of participants mentioned that they worked closely with an otorhinolaryngologist to assist in the vestibular rehabilitation, $18 \%$ reported to work with neurologists, $8 \%$ of them did not work with other professionals, and $3 \%$ of the participants worked with the physiotherapists. 
Subjective Assessment Tools

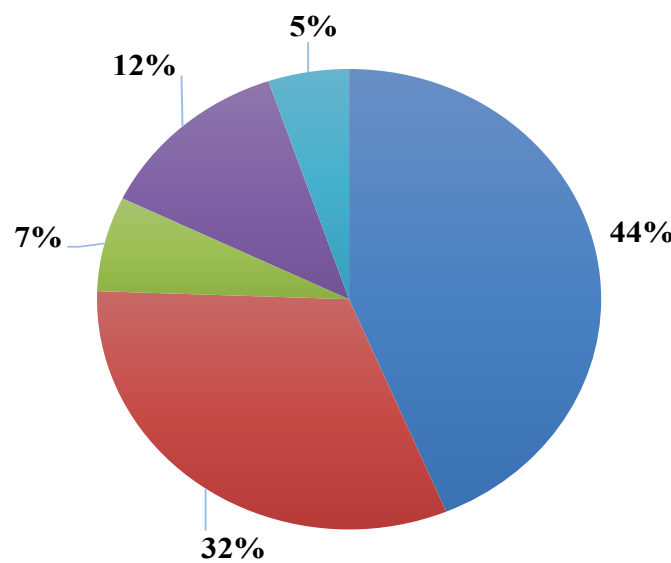

Dizziness

Handicap Inventory

(DHI)

Vertigo Handicap

Questionnaire(VHQ)

- Vestibular Disorders

Activities of Daily

Living (VADL)

- Vertigo Symptom

Scale (VSS)

- Others

Figure 1: Percentage of audiologists indicating subjective assessment tools.

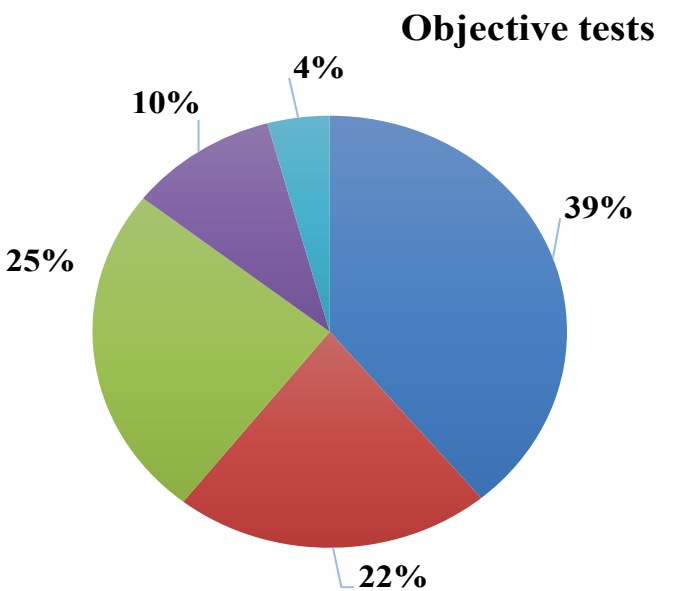

- Cervical Vestibular

Evoked Myogenic

Potentials (cVEMP)

Videonystagmography (VNG)

Electronystagmography (ENG)

- Video Head Impulse Test (vHIT)

Figure 2: Percentage of audiologists indicating objective tests of vestibular assessment.

\section{Components of vestibular test battery}

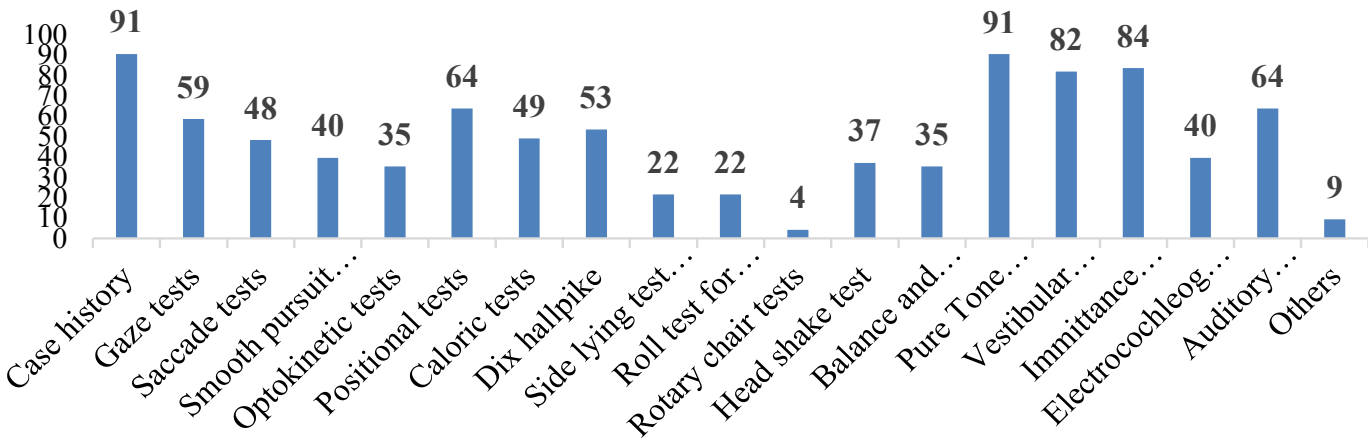

Figure 3: Percentage of audiologists indicating tests used in vestibular test battery.

Rehabilitation Strategies: The types of treatment strategies used by audiologists in India have been shown in figure 4 . A majority $(75 \%)$ of the participants in the current study reported the use of balance exercises, $54 \%$ reported the use of habituation exercise, $43 \%$ used canalith repositioning maneuvers, $34 \%$ used gaze stabilization exercises, and $5 \%$ of the participants used other strategies as indicated in figure 4 . About 21 participants out of 116 reported that they use a combination of two strategies, which included habituation and balance exercises, and 20 participants used a combination of three or more strategies for vestibular rehabilitation. For effective management, the clinician must demonstrate the appropriate steps involved in a particular vestibular rehabilitation strategy. In this regard, we asked our participants how often they demonstrated vestibular rehabilitation strategies to their patients. Nearly $47 \%$ of our participants "always" demonstrated rehabilitation exercises/maneuvers to their patients, $25 \%$ demonstrated 'sometimes', $20 \%$ of the participants preferred 'not to 


\section{Rehabilitation trends}

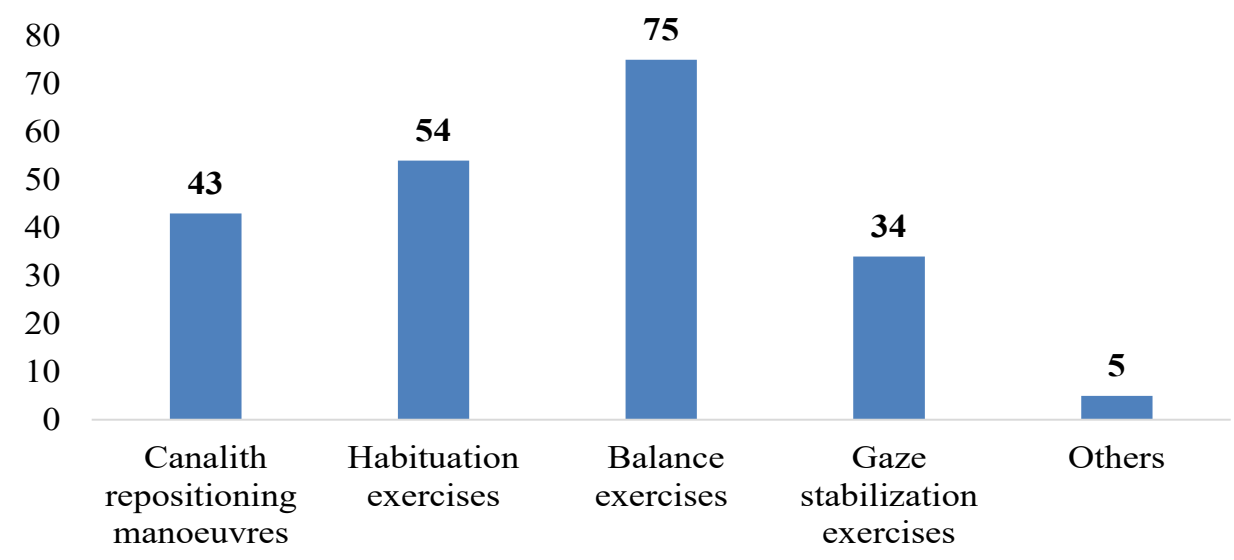

Figure 4: Percentage of rehabilitation procedures reported by participants of the survey.

say,' and $8 \%$ of our participants did not demonstrate exercises to their patients. $81 \%$ of the participants agreed that demonstrating rehabilitation exercises/maneuvers to their patients would result in a greater impact on vestibular rehabilitation, $15 \%$ were neutral, $3 \%$ of the participants reported that they did not know. These responses are similar to the study by Nelson et al. ${ }^{6}$, which suggests that most of the professionals demonstrate exercises and it. While recommending vestibular rehabilitation, $61 \%$ of the participants 'always' recommended a home plan for their patients, $29 \%$ recommended sometimes, $7 \%$ preferred 'not to say', and $3 \%$ of the participants did not recommend a home plan.

Rehabilitation monitoring: $46 \%$ of the participants reported that they used the Dizziness Handicap Inventory $(\mathrm{DHI})^{9}$ to monitor the progress of their patients with vestibular disorders, $26 \%$ of our participants relied on presence/ absence of spontaneous/positional nystagmus, $20 \%$ of participants reported the use of all the mentioned methods (DHI, Presence/absence of spontaneous/ positional nystagmus and Disability Rating Scale). However, almost $19 \%$ of the participants reported that they did not monitor the progress, and $4 \%$ reported other methods. Out of all the participants of the survey who performed vestibular rehabilitation, $55 \%$ of the participants always recommended follow-up to their patients, $29 \%$ recommended 'sometimes', $9 \%$ preferred not to say, and $4 \%$ did not recommend follow-up.

Barriers to practice; $52 \%$ of the participants reported to be 'confident' in performing the vestibular rehabilitation, $7 \%$ were 'highly confident,' nearly $41 \%$ were 'not confident' in managing vestibular disorders and reported the lack of training, and experience to be the reason. Several other barriers such as lack of equipment, funding, training, experience, and fewer referrals served as challenges to the efficient practice of vestibular rehabilitation.

\section{CONCLUSION}

Vestibular disorders have grave consequences on the physical (in terms of increased fall risk), psychiatric- psychological (such as anxiety, depression), and cognitive domain, and thus affecting the overall quality of life. As the incidence of vestibular disorders continues to rise, the role of the audiologists in assessing and providing effective intervention has become increasingly important. To our knowledge, the current study is the first of its kind to investigate the practice patterns of Indian audiologists towards vestibular assessment and management. The findings of the current study indicate a large variability between the opinions and practices of vestibular assessment and management in India. Like any other developing nation, India has challenges of its own. Proper planning in terms of resource allocation is needed to address the barriers in access to instrumentation, and limited workforce.

\section{CONFLICT OF INTEREST}

The authors declares no conflict of interest

\section{REFERENCES}

1. Neuhauser, HK. The epidemiology of dizziness and vertigo. Neuro-Otology. 2016;1: 67-82.

2. Koo JW, Chang MY, Woo S, Kim S, Cho YS. Prevalence of vestibular dysfunction and associated factors in South Korea. BMJ Open. 2015;5; e00822-4.

3. Shumway CA, Horak FB. Rehabilitation Strategies for Patients with Vestibular Deficits. Neurologic Clinics. 2004;8: 441-57.

4. Brandt T, Strupp M. General vestibular testing. Clinical Neurophysiology. 2005; 116: 406-26.

5. Gans RE. Vestibular Diagnosis and Rehabilitation: Science and Clinical Applications. Seminars in Hearing. 2002;23: 105-106.

6. Nelson MD, Akin FW, Riska KM, Andresen K, Mondelli SS. Vestibular Assessment and Rehabilitation: Ten-Year Survey Trends of Audiologists' Opinions and Practice. J Am Academy of Audiol. 2006;27: 126-40.

7. Seedat T, Khoza-Shangase K, Sebothoma B. Vestibular assessment and management in adults: current practice by South African audiologists. Hearing, Balance and Communication. 2018;16: 88-100. 
8. Meldrum D, Burrows L, Cakrt O, Kerkeni H, Lopez C, Jahn, $\mathrm{K}$. Vestibular rehabilitation in Europe: a survey of clinical and research practice. J Neurol. 2020; 267; 24-35.

9. Treleaven J. Dizziness handicap inventory (DHI). Australian J Physiother. 2006;1: 1-6.

10. Yardley L, Putman J. Quantitative analysis of factors contributing to handicap and distress in vertiginous patients: a questionnaire study. Clinical Otolaryngol \& Allied Sci. 2007; 17: 231-6.
11. Cohen HS, Kimball KT. Development of the vestibular disorders activities of daily living scale. Arch of Otolaryngol-Head \& Neck Surg. 2006;126: 881-7.

12. Yardley L, Masson E, Verschuur C, Haacke N, Luxon L. Symptoms, anxiety and handicap in dizzy patients: development of the vertigo symptom scale. J Psychosomatic Res. 1982;36: 731-41. 